\title{
Registro de Struthiopterolichus sculpturatus e Struthiolipeurus rheae em Struthio camelus no Rio Grande do Sul, Brasil
}

\author{
Record of Struthiopterolichus sculpturatus and Struthiolipeurus rheae in Struthio camelus in the Rio \\ Grande do Sul State, Brazil
}

\author{
Tatiana Cheuiche Pesenti ${ }^{{ }^{*}}$ Diego Silva da Silva ${ }^{\mathrm{I}}$ Leonardo Lopes Bertacco ${ }^{\mathrm{II}}$ \\ João Guilherme Werner Brum ${ }^{I}$ Gertrud Müller ${ }^{\mathrm{I}}$
}

\begin{abstract}
Avestruzes são ratitas originárias da África, sendo encontradas no Brasil, principalmente, em criatórios comerciais, os quais vêm se expandido no país devido ao potencial reprodutivo e alto valor comercial de seus produtos como carne, couro, gordura, plumas e ovos. Porém, as parasitoses são causas de grandes prejuízos econômicos e morte nesses criatórios. Em consequência disso, objetivou-se identificar os ectoparasitos que acometem avestruzes de criatórios comerciais no Rio Grande do Sul. Para tanto, foram coletadas, ao acaso, amostras de plumas e cabeças de 34 avestruzes, durante o abate, no frigorifico de Júlio de Castilhos, Rio Grande do Sul (RS). As amostras de plumas, as cabeças e as fossas nasais foram lavadas, individualmente, com água contendo detergente. Em seguida, o material foi tamisado em malha $150 \mu \mathrm{m}$ e examinado ao estereomicroscópio para coleta dos ectoparasitos. Nas plumas, foram encontrados piolhos e ácaros, os quais foram clarificados em salicilato de metila e lactofenol, respectivamente, e identificados como Struthiolipeurus rheae e Struthiopterolichus sculpturatus. Este é o primeiro registro de ocorrência desses ectoparasitos em avestruzes no Rio Grande do Sul.
\end{abstract}

Palavras-chave: avestruz, Struthiolipeurus rheae, Struthiopterolichus sculpturatus, piolho, ácaro.

\section{ABSTRACT}

Ostriches are originated from Africa, and in Brazil they are mostly found in commercial farms which are developing in Brazil due to the great reproductive potential and the great economic value of its products that include meat, leather, feathers and eggs. However, parasitosis causes death of the birds and economic failure. Therefore, the aim of this research was to identify these ectoparasites that infect ostriches from commercial farms in the Rio Grande do Sul state, Brazil. Feathers and heads were collected randomly from 34 ostriches slaughtered in the city of Julio de Castilhos, RS. The feathers, heads and nasal cavity were individually cleaned in water with detergent. Following this, the material was sieved in a $150 \mu \mathrm{m}$ sieve and examined by stereomicroscope to search for ectoparasites search. Lice and mites were found in the feathers. The lices were clarified using metil salicilate and the mites by lactophenol. The lices were identified as Struthiolipeurus rheae and the mites as Struthiopterolichus sculpturatus. This is the first description of these arthropods in ostriches in Rio Grande do Sul.

Key words: ostrich, Struthiolipeurus rheae, Struthiopterolichus sculpturatus, lice, mite.

Ectoparasitos podem ser encontrados infestando as plumas de avestruzes, provocando irritação e prurido, causados pela movimentação e atividade alimentar no corpo do hospedeiro, e ocasionando perda excessiva de plumas (PRICE \& GRAHAM, 1997). Além disso, podem provocar estresse, predisposição das aves a infecções secundárias e distúrbios gastrintestinais, podendo levar a perdas significativas no plantel (COOPER, 2005).

PONCE GORDO et al. (2002) identificaram, na Espanha, Struthiolipeurus rheae (Harrison, 1916) nos avestruzes e, $\boldsymbol{S}$. rheae e $\boldsymbol{S}$. nandu nas emas procedentes de criatórios de vários países da Europa. No Chile, VERA et al. (2007) fizeram o primeiro registro

ILaboratório de Parasitologia de Animais Silvestres, Departamento de Microbiologia e Parasitologia, Universidade Federal de Pelotas (UFPel), 96010-900, Pelotas, RS, Brasil. E-mail: tatianapesenti@yahoo.com.br. *Autor para correspondência.

"Médico Veterinário, Associação de Produtores de Avestruz do Rio Grande do Sul (CPARS), Porto Alegre, RS, Brasil. 
do piolho $S$. rheae em avestruzes. No Brasil, o encontro de piolhos em ratitas foi citado pela primeira vez por SILVA et al. (2004), em Sorocaba, São Paulo (SP), que identificaram Struthiolipeurus rheae e $S$. nandu em $R$. americana. RIBEIRO et al. (2004a), em Porto Alegre, Rio Grande do Sul (RS), fizeram o primeiro registro de S. struthionis em avestruzes para o Estado do RS e em São Paulo S. struthionis foi encontrado na mesma espécie por VALIM et al. (2005). SINKOC et al. (2005) identificaram $\boldsymbol{S}$. rheae em emas provenientes de criatórios do RS e Minas Gerais (MG), sendo a primeira ocorrência desse piolho para esses Estados. MENEZES et al. (2006) encontraram S. rhea nas plumas de avestruzes de um criatório comercial em Três Rios, Rio de Janeiro (RJ), e ALMEIDA et al. (2006) constataram a ocorrência de $S$. rheae em avestruzes da região semiárida de Irecê, Bahia (BA).

Quanto aos ácaros, PONCE GORDO et al. (2002), na Espanha, encontraram avestruzes infestados por Dermoglyphus pachycnemis e Gabucinia bicaudata, já na Austrália HALLIDAY (2006) registrou a ocorrência de Struthiopterolichus bicaudatus em avestruzes. No Brasil, RIBEIRO et al. (2004b) encontraram ácaros nas penas de avestruzes de uma criação em Glorinha, RS, identificados como $\boldsymbol{G}$. bicaudata, cuja citação foi a primeira para o Estado. FACCINI et al. (2006) amostraram avestruzes para pesquisa de ectoparasitos em uma fazenda de Caratinga, MG, identificando S. bicaudatus. ALMEIDA et al. (2006) encontraram avestruzes do Município de Irecê, BA, infestados pelo ácaro $\mathbf{G}$ bicaudata. Em um criatório na região da Baixada Fluminense, RJ, MATTOS et al. (2007) coletaram ácaros nas plumas das asas de uma avestruz que apresentava áreas desplumadas na região das asas e irritação na pele, os quais foram identificados em relação à Struthiopterolichus sculpturatus (Dubinin, 1955).

A expansão da estrutiocultura no Brasil e o aparecimento das parasitoses nos criatórios levaram ao desenvolvimento deste trabalho, cujo objetivo foi identificar os ectoparasitos de avestruzes do Rio Grande do Sul.

Cinco amostras de plumas (em média $250 \mathrm{~g}$ cada) e cabeças de 34 avestruzes foram coletadas, ao acaso, durante o abate no frigorífico, em Júlio de Castilhos, RS, em duas ocasiões, uma no mês de março e outra em outubro de 2007. O material foi acondicionado em sacos plásticos, bem fechados, e encaminhado ao Laboratório de Parasitologia de Animais Silvestres do DEMP-IB-UFPel (Departamento de Microbiologia e Parasitologia do Instituto de Biologia da Universidade Federal de Pelotas) para análise. Cada amostra de pluma foi lavada em recipiente contendo água com detergente (detergente líquido no proporção de $10 \mathrm{~L}$ de água para $50 \mathrm{ml}$ de detergente). A superfície externa de cada cabeça foi inspecionada e também lavada em água contendo detergente (na mesma proporção utilizada nas plumas). Em ambos os casos, a água resultante da lavagem foi tamisada em malha $150 \mu \mathrm{m}$, e o material resultante foi examinado ao estereomicroscópio para coleta de ectoparasitos, os quais foram acondicionados em frascos com álcool $70^{\circ} \mathrm{GL}$ para posterior identificação. As fossas nasais foram examinadas fazendo-se um corte a partir das narinas até o orifício externo do ouvido do lado correspondente; o corte também foi feito do outro lado, rebatendo-se a parte superior da cabeça para trás até formar um ângulo reto com a parte inferior. $\mathrm{O}$ interior das fossas nasais foi lavado, o material foi tamisado em malha $150 \mu \mathrm{m}$ e as mucosas foram examinadas ao estereomicroscópio. Os ácaros encontrados foram clarificados em lactofenol, montados em lâminas permanentes com bálsamo do Canadá e identificados segundo ANDRÉ (1960). Os piolhos foram clarificados em salicilato de metila, montados em lâminas permanentes com bálsamo do Canadá e identificados conforme WEISBROTH \& SEELIG (1974). Os exemplares foram catalogados e depositados na coleção de artrópodes do Laboratório de Parasitologia de Animais Silvestres do Instituto de Biologia da Universidade Federal de Pelotas.

De acordo com o verificado, não foram encontrados ectoparasitos na superfície externa da cabeça, bem como nas fossas nasais das avestruzes.

Das plumas foram coletados espécimes de piolhos da Família Philopteridae, espécie Struthiolipeurus rheae (Figuras 1A e 1B), caracterizando o primeiro registro de ocorrência dessa espécie em avestruzes para o Estado do RS. Essa espécie já havia sido registrada em avestruzes, em outros Estados brasileiros (MENEZES et al., 2006; ALMEIDA et al., 2006), bem como em emas (SINKOC et al., 2005), e na Europa e no Chile somente em avestruzes (PONCE GORDO et al., 2002; VERA et al., 2005), respectivamente. Além disso, nas plumas também foram encontrados ácaros pertencentes à Ordem Astigmata, Família Pterolichidae, Struthiopterolichus sculpturatus (Figuras 1C e 1D), fazendo-se, também, o primeiro registro de ocorrência dessa espécie para o RS. No Brasil, esse ácaro havia sido registrado apenas para o Rio de Janeiro, (MATTOS et al., 2007), nas plumas de avestruzes.

Por meio do registro de ocorrência do piolho S. rheae e do ácaro S. sculpturatus em avestruzes de criatórios comerciais do RS, alerta-se a classe veterinária e os criadores para necessidade de vigilância e controle desses ectoparasitos, evitando futuros prejuízos nos plantéis. 


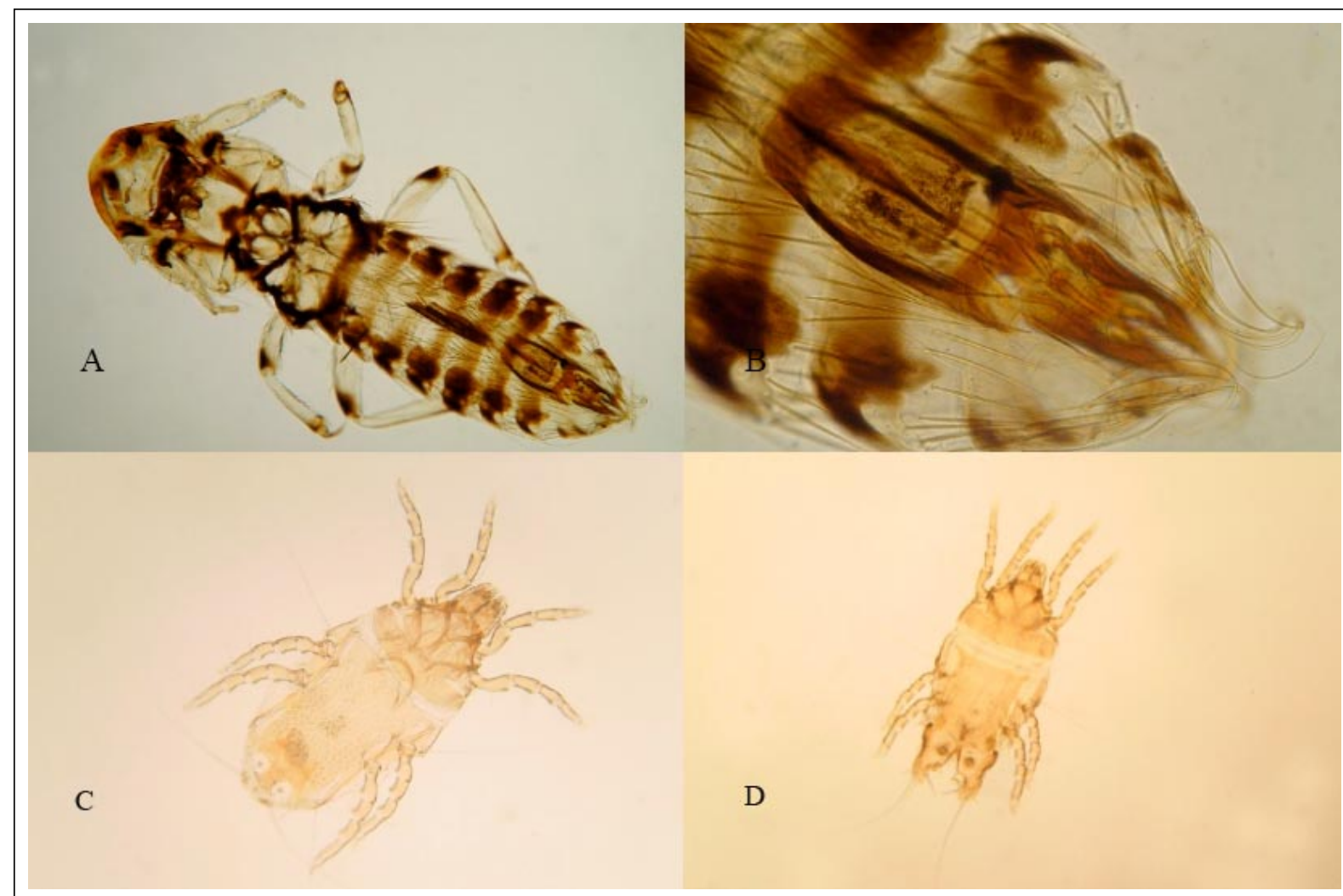

Figura 1 - (A) Macho de Struthiolipeurus rheae, aumento de 5x. (B) Genitália do macho de S. rheae, aumento de 20x. (C) Fêmea de Struthiopterolichus sculpturatus, aumento de 10x. (D) Macho de S. sculpturatus, aumento de 10x.

\section{REFERÊNCIAS}

ALMEIDA, M.A.O. et al. Ectoparasitos em avestruzes (Struthio camelus) criadas no semi-árido baiano. In: CONGRESSO BRASILEIRO DE PARASITOLOGIA VETERINÁRIA, 14., 2006, Ribeirão Preto, SP . Anais... Ribeirão Preto: Colégio Brasileiro de Parasitologia Veterinária, 2006. 428p. p.198.

ANDRÉ, M. Sarcoptides plumicoles parasites des autruches. Acarologia, v.2, p.556-567, 1960.

COOPER, R.G. Bacterial, fungal and parasitic infections in the ostrich (Struthio camelus var. domesticus). Animal Science Journal, v.76, p. 97-106, 2005. Disponível em: www.scielo.br/ s c i eloOrg/p h p/articleXML.php? pid=S 0102 $09352008000500036 \&$ lang=en. Acesso em: 05 mai 2007.

FACCINI, J.L.H. et al. Occurence of Struthiopterolichus bicaudatus (Acari; Pterolichidae) in southeastern Brazil. Arquivo Brasileiro de Medicina Veterinária e Zootecnia, v.58, n.5, 2006. Disponível em: www.scielo.br/ scielo.php?pid=S0102-9352006000500039\&script $=$ sci_arttext. Acesso em: 30 fev 2007. doi: 10.1590/S010209352006000500039 .

HALLIDAY, R.B. Struthiopterolichus sculpturatus (Gervais) (Acari: Pterolichidae): a feather mite pest of the ostrich in Australia. Australian Veterinary Journal, v.8, n.1\&2, p. 68-69, 2006.
MATTOS, D.G. et al. Registro de ácaros em avestruz no estado do Rio de Janeiro. Arquivo Brasileiro de Medicina Veterinária e Zootecnia, v.59, n.2, p.536538, 2007. Disponível em: www.scielo.br/scieloOrg/php/ articleXML.php?pid=S0102-09352008000500036\&lang=en. Acesso em: 07 mai 2007.

MENEZES, R. et al. Struthiolipeurus rheae (Phthiraptera: Philopteridae) identificados em avestruzes (Struthio camelus) pertencentes a uma criação comercial localizada no município de Três Rios, RJ. In: CONGRESSO BRASILEIRO DE PARASOTOLOGIA VETERINÁRIA, 14., 2006, Ribeirão Preto, SP. Anais... Ribeirão Preto: Colégio Brasileiro de Parasitologia Veterinária, 2006. 428p. p.204.

PONCE GORDO, F. et al. Parasites from farmed ostriches (Struthio camelus) and rheas (Rhea americana) in Europe. Veterinary Record, v.107, p.137-160, 2002. Disponível em: www.linkinghub.elsevier.com/retrieve/pii. Acesso em: $22 \mathrm{mar}$ 2007. doi: 10.1016/S0304-4017(02)00104-8.

PRICE, M.A.; GRAHAM, O.H. Chewing and sucking lice as parasites of mammals and birds. USDA, ARS, Techical Bulletin, n. 1849,257 p, 1997.

RIBEIRO, V.L.S. et al. Ocorrência de Struthiolipeurus struthionis (Gervais, 1844) (Phthiraptera: Philopteridae) em Struthio camelus Linnaeus, 1758 (Struthioniformes: Struthionidae) no Rio Grande do Sul, Brasil. In: CONGRESSO 
BRASILEIRO DE PARASITOLOGIA VETERINÁRIA, 13., 2004, Ouro Preto, MG. Anais... Ouro Preto: Colégio Brasileiro de Parasitologia Veterinária, 2004a. V.13. 396p. p.332.

RIBEIRO, V.L.S. et al. Ocorrência de Gabucinia bicaudata (Acari: Gabuciniidae) em Struthio camelus (Struthioniformes: Struthionidae) no Rio Grande do Sul. In: CONGRESSO BRASILEIRO DE PARASITOLOGIA VETERINÁRIA, 13. 2004, Ouro Preto, MG. Anais... Ouro Preto: Colégio Brasileiro de Parasitologia Veterinária, 2004b. V.13. 396p. p.3346.

SILVA. S.O. et al. Malófagos parasitas de aves campestres cativas do Zoológico Municipal Quinzinho de Barros, Sorocaba, Estado de São Paulo, Brasil. Entomologia y Vectores, v.11, n.2, p.333-339, 2004. Disponível em: www.phthiraptera.org/ publications/46943.pdf. Acesso em: 13 jun 2006.

SINKOC, A.L. et al. Ocorrência de Struthiolipeurus rheae (Phthiraptera: Ischnocera: Philopteridae) em Rhea americana (Rheiformes: Rheidae) no Brasil. Arquivos do Instituto Biológico, v.72, n.4, p.535-538, 2005. Disponível em:
www.biologico.sp.gov.br/docs/arq/v72_4/sinkoc.PDF. Acesso em: 17 jun 2006.

VALIM, M.P. et al. Malófagos (Phthiraptera) recolhidos de aves silvestres no zoológico de São Paulo, SP, Brasil. Revista Brasileira de Entomologia, v.49, n.4, p.584-587, 2005. Disponível em: www.scielo.br/scieloOrg/php/ articleXML.php?pid=S0085-56262005000400021\&lang=en. Acesso em: 24 mar 2006. doi: 10.1590/S008556262005000400021 .

VERA, K. et al. Detección de la presencia de endoparasitos y ectoparasitos de avestruces (Struthio camelus) em criaderos de la Sexta Región y Región Metropolitana. Boletín Veterinario oficial, BVO, n.8, I semestre 2007. Disponível em: www2.sag.gob.cl/pecuaria/bvo. Online. Acesso em 08 de agosto. 2007.

WEISBROTH, S.H.; SEELIG, A.W. Struthiolipeurus rhea (Mallophaga: Philopteridae) an ectoparasite of the commom rhea (Rhea americana). J ournal of Parasitology, v.60, n.5, p.892-894, 1974. 